Selected papers from the International Scientific and Practical Conference "Basic Science for Practical Medicine - 2021", 15-18 September 2021, Elbrus v., Russia, http://uniid.kbsu.ru/medicine2021

Original article

\title{
Hemocompatibility and cytotoxicity of small-diameter bioabsorbable tissue-engineered vascular grafts depending on anti-thrombogenic and antimicrobial coating
}

\author{
Eugenia O. Krivkina, Elena A. Velikanova, Eugenia A. Senokosova, Maryam Yu. Khanova, Tatyana V. Glushkova, \\ Larisa V. Antonova, Leonid S. Barbarash
}

Research Institute for Complex Issues of Cardiovascular Diseases, Kemerovo, Russia

Received 1 October 2021, Revised 26 November 2021, Accepted 10 December 2021

(C) 2021, Russian Open Medical Journal

Abstract: Anti-thrombogenic and antimicrobial coatings of polymer grafts constitute a promising approach to preventing infection and thrombosis of vascular grafts.

The objective was to study the hemocompatibility and cytotoxicity of PHBV/PCL grafts with iloprost and amphiphilic coating.

Material and Methods - Polymer matrices were manufactured by electrospinning a mixture of poly(3-hydroxybutyrate-co-3hydroxyvalerate) (PHBV) and poly( $\varepsilon$-caprolactone) (PCL) polymers. Several matrices were modified by complexation between polyvinylpyrrolidone (PVP) and cationic amphiphile and/or iloprost. The amphiphile was covalently cross-linked to the surface of other PHBV/PCL matrices. Unmodified PHBV/PCL matrices were used as the control group. Hemocompatibility and cytotoxicity of scaffolds before and after the modification were evaluated.

Results - The hemocompatibility assessment revealed that hemolysis degree did not exceed normal values in all types of matrices. The PHBV/PCL/PVP matrices had increased platelet aggregation on the surface of the grafts. Subsequent addition of iloprost and amphiphile resulted in a sevenfold reduction of platelet aggregation. In PHBV/PCL/PVP matrices, the degree of platelet adhesion increased without changing the platelet deformation index values. lloprost and amphiphilic coating of PHBV/PCL/PVP matrices diminished the number of adhered platelets and platelet deformation index by 1.5 times. The amphiphile, covalently cross-linked to PHBV/PCL matrices, caused a negative effect on the platelet adhesion, aggregation, and deformation index values. Evaluation of cytotoxicity of PHBV/PCL/PVP matrices, coated with iloprost and/or cationic amphiphile, demonstrated a slight decline in the rates of cell growth and proliferation after three days. Moreover, after three days, cell deaths and a sharp drop in the cell index values were noted in PHBV/PCL matrices with covalently crosslinked amphiphile.

Conclusion - lloprost and amphiphilic coating of PHBV/PCL grafts has increased their hemocompatibility. Also, there were no signs of cytotoxicity while using the complexation technique. However, covalently cross-linked amphiphile caused an increase in the cytotoxicity of matrices, which may have been indicative of the negative effect observed in this type of surface modification.

Keywords: vascular prostheses, hemocompatibility, electrospinning, cationic amphiphiles, cytotoxicity.

Cite as Krivkina EO, Velikanova EA, Senokosova EA, Khanova MYu, Glushkova TV, Antonova LV, Barbarash LS. Hemocompatibility and cytotoxicity of smalldiameter bioabsorbable tissue-engineered vascular grafts depending on anti-thrombogenic and antimicrobial coating. Russian Open Medical Journal 2021; 10: e0423.

Correspondence to Eugenia O. Krivkina. Address: 6 Sosnovyi Blvd, Kemerovo 650002, Russia. Phone: +79089466639. E-mail: leonora92@mail.ru.

\section{Introduction}

Tissue engineering is among the most promising fields of vascular graft research that has the potential to create a vascular graft with a tubular matrix made of natural and/or synthetic bioabsorbable and biocompatible polymers. Such matrix is a scaffold that can be subsequently populated by autologous cells in vitro or in situ [1].

Despite widespread use of polymer grafts in cardiovascular surgery, the graft infection remains a serious problematic issue with severe complications and high mortality rates [2]. It is difficult to establish specific etiology of graft infections, since they are usually multifactorial [3]. Bacteria may originate from an exogenous or endogenous source (bacteremia) $[4,5]$. Despite the sterile conditions in the course of implantation, about $20 \%$ of grafts become infected, increasing the percentage of subsequent occlusion to $27 \%$ [6]. Pathogenic bacteria are able to colonize the graft surface, forming a protective biofilm that increases their antibiotic resistance and host defense mechanisms, which may lead to a vascular graft replacement $[7,8]$. The biofilm formation could be prevented by replacing the usual graft materials with antimicrobial and biocompatible polymers, or by an additional antimicrobial treatment $[9,10]$. Taking into account the declining 
effectiveness of antibacterial medicines due to the emergence of bacteria with multiple drug resistance, the development of vascular grafts with antimicrobial coatings that have a low chance of inducing antibiotic resistance becomes very relevant. Immobilizing the antibacterial preparation directly on the graft surface would solve the problem of local drug delivery, and ensure the proper effect on microorganisms prior to the biofilm formation.

In the last decade, hydrogels have become widespread in cardiovascular surgery due to their ability to deliver pharmaceutical drugs and release them over long periods of time [11]. Hydrogels are represented by the three-dimensional polymer networks obtained from water-soluble or bioabsorbable natural/synthetic polymers with antibacterial agent bound to the polymer matrix by non-covalent interactions.

Cationic amphiphiles are single or multiple positively charged molecules with lipophilic fragments which can potentially resolve the problem of drug resistance and help creating an effective antimicrobial coating $[12,13]$. These compounds are synthetic analogs of natural cationic antimicrobial peptides. They can cause disruption of the transmembrane potential, cytoplasmic content leakage, and, ultimately, cell death [12]. Most of these compounds are highly effective against both gram-positive and gram-negative bacteria (including multidrug-resistant bacteria). The amphiphiles that destroy bacterial membranes and, simultaneously, other targets, can significantly reduce the likelihood of bacteria developing resistance to such compounds. High stability of these compounds, even in physiological fluids, in combination with their low cost, makes them the most promising candidates for the role of low molecular weight modifiers of polymer materials.

An important issue in manufacturing the tissue-engineered vascular grafts is ensuring the porosity of the graft wall, since it is required to provide full migration of cells from the bloodstream and surrounding tissues, cell proliferation, and differentiation. However, such porous surface could lead to the thrombus formation. Consequently, additional modification to the surface of grafts with antiplatelet and anticoagulant agents could prevent the thrombus formation after graft implantation.

Moreover, it is also necessary to take into account the cytotoxicity of drugs and binding agents used for antimicrobial and anti-thrombogenic coatings in the course of the manufacturing process.

\section{Material and Methods}

\section{Manufacturing the vascular grafts}

Small-diameter vascular grafts $(4 \mathrm{~mm})$ were produced by emulsion electrospinning a mixture of $2 \%$ poly(3-hydroxybutyrateco-3-hydroxyvalerate) (PHBV, Sigma-Aldrich, USA) and $12 \%$ poly $(\varepsilon-$ caprolactone) (PCL, Sigma-Aldrich, USA); 1,1,1,3,3,3-hexafluoro-2propanol (Sigma-Aldrich, USA) was used as a solvent. Electrospinning was performed using a Nanon-01A nanofiber electrospinning system (MECC, Inc., Japan) at a voltage of $20 \mathrm{kV}$, a solution feed rate of $0.5 \mathrm{~mL} / \mathrm{h}$, a collector rotation speed of 1000 $\mathrm{rpm}$, a needle movement speed of $60 \mathrm{~mm} / \mathrm{s}$, and $150 \mathrm{~mm}$ distance from the needle to the collector.

\section{Anti-thrombogenic and antimicrobial coatings of vascular grafts}

Surface modification of the manufactured polymer scaffolds was performed in two different ways: some of the manufactured vascular grafts were modified sensu our own original technique of forming a polyvinylpyrrolidone (PVP) hydrogel coating on the inner surface. To achieve this, the grafts were placed in argon-filled glass test tubes, then sealed with parafilm and irradiated at a dosage of $15 \mathrm{kGy}$ using an ILU-10 accelerator with energy range up to $5 \mathrm{MeV}$ and beam power of $50 \mathrm{~kW}$ (Budker Institute of Nuclear Physics at the Siberian Branch of Russian Academy of Sciences). Hence, sterilization of vascular grafts took place along with their modification. Consequently, all further manipulations were carried out under sterile conditions. Then, platelet antiaggregant, iloprost (Ilo), and/or cationic amphiphile, 1,5-bis-(4-tetradecyl-1,4diazoniabicyclo [2.2.2] octan-1-yl) pentane tetrabromide (A), with antimicrobial and antiviral properties were added to the PVP hydrogel coating via employing the complexation technique [14].

All other grafts were modified by covalent crosslinking, implemented via immobilization of the amphiphile on the surface of nonwoven matrix due to activation of carboxyl groups in the polymer by a water-soluble carbodiimide, followed by the treatment with a peptide solution.

\section{Hemocompatibility analysis}

Hemocompatibility of grafts was assessed via hemolysis analysis and platelet adhesion, aggregation, and deformation index.

\section{Hemolysis}

The assessment of the hemolysis degree after the contact with the surface of polymer samples was conducted sensu the ISO 10993.4 standards: $3.8 \%$ sodium citrate was added to the fresh donor blood in the ratio of 1:9. Five samples of $25 \mathrm{~cm}^{2}$ each were placed in weighing cups containing $10 \mathrm{~mL}$ of physiological solution, and were incubated for $120 \mathrm{~min}$ at $37^{\circ} \mathrm{C}$. Saline and distilled water were used as positive and negative controls, respectively. After two hours of incubation, $200 \mu \mathrm{L}$ of citrated blood was added to each cup for further incubation during $60 \mathrm{~min}$ at $37^{\circ} \mathrm{C}$. Then, the polymer samples were transferred from the cups into test tubes and centrifuged for $10 \mathrm{~min}$ at $2,800 \mathrm{rpm}$ to precipitate the remaining cells. Optical density of obtained supernatants was measured using the GENESYS 6 spectrophotometer (Thermo Scientific, USA) at a wavelength of $545 \mathrm{~nm}$.

The percentage of hemolysis $(\mathrm{H})$ was calculated via the following equation $[15,16]$ :

$$
H(\%)=\frac{D_{t}-D_{n e}}{D_{p e}-D_{n e}} \times 100 \%
$$

where, $D_{t}$ is the optical density of incubated sample; $D_{n e}$ is the optical density of the positive control; $D_{\text {pe }}$ is the optical density of the sample with $100 \%$ hemolysis.

Zero hemolysis value was defined as the mean optical density of saline-diluted blood (positive control); $100 \%$ hemolysis value was defined as the mean optical density of blood diluted with distilled water: $\mathrm{D}_{\mathrm{pe}}=0.279$ (negative control).

\section{Platelet aggregation}

The study was carried out in accordance with the ISO 10993.4 standard procedures. To obtain platelet-rich plasma (PRP), citrated blood was centrifuged for $10 \mathrm{~min}$ at $1,000 \mathrm{rpm}$. Platelet-poor plasma (PPP) was obtained by repeated centrifugation of PRP for 
20 min at 4,000 rpm. The centrifuge was properly calibrated. Intact PRP was used as a positive control. The measurements were performed in a spontaneous mode without aggregation inductors. $\mathrm{CaCl}_{2}(0.025 \mathrm{~mol} / \mathrm{L})$ was used to restore the level of $\mathrm{Ca}^{+2}$ in citrated blood. The reagent mixture contained $250 \mu \mathrm{L}$ of PRP and $25 \mu \mathrm{L}$ of $\mathrm{CaCl}_{2}$. The contact time of the test samples with PRP was 3 min, after which the measurements were made. The platelet maximum aggregation rate was measured by the semi-automated 4-channel platelet aggregometer APACT 4004 (LABiTec, Germany).

\section{Platelet adhesion and platelet deformation index}

Samples of polymer matrices $(n=5), 0.5 \mathrm{~cm}^{2}$ each, were incubated for 2 hours at $37{ }^{\circ} \mathrm{C}$ in $300 \mu \mathrm{L}$ of PRP obtained from fresh citrated donor blood by centrifugation for $10 \mathrm{~min}$ at 1,200 rpm. Next, test samples were rinsed with phosphate-buffered saline (PBS, $\mathrm{pH}-7.4)$ in order to remove non-adherent plasma components. Then the samples were fixed in a $2 \%$ glutaraldehyde solution for 24 hours, rinsed with PBS and dehydrated in alcohols with concentrations ranging from $30 \%$ to $100 \%$ for 15 minutes each, followed by drying at a room temperature. Later on, samples were mounted on special tables by means of the carbon tape to form a conductive (Au/Pd) coating, using EM ACE200 vacuum coater (Leica Mikrosysteme $\mathrm{GmbH}$, Austria). For analysis, we randomly selected 8 samples. The adhesion ability of their surfaces was assessed with by means of the platelet count (platelet per 1 $\mathrm{mm}^{2}$ ), predominant platelet type on the surface, and also by platelet deformation index (PDI), calculated by the following formula [15-18]:
PDI = (number of type I platelets $\times 1+$ number of type II platelets $\times 2+$ number of type III platelets $\times 3+$ number of type IV platelets $\times 4+$ number of type $V$ platelets $\times 5$ ) / total number of platelets.
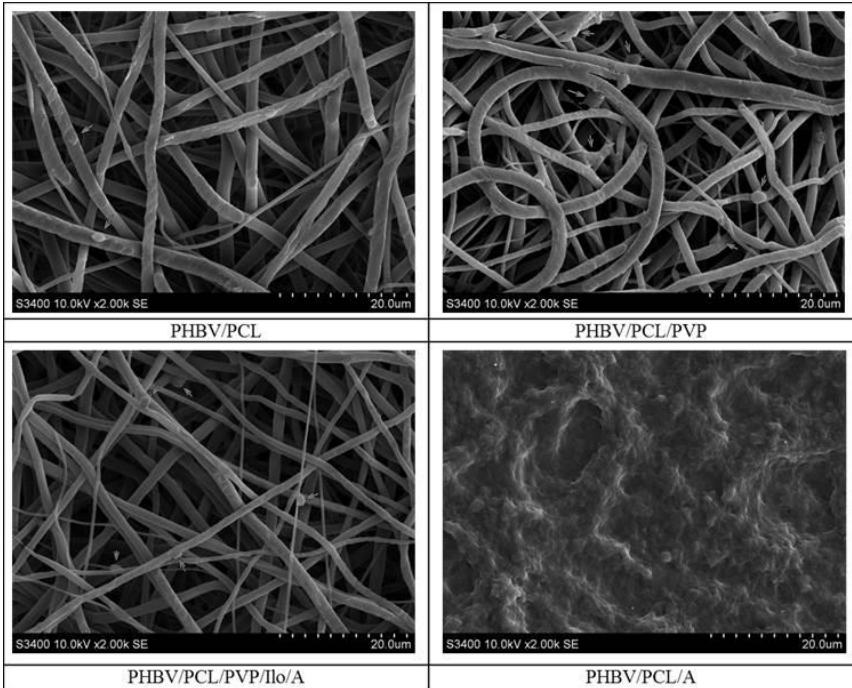

Figure 1. Surfaces of PHBV/PCL matrices after the contact with PRP (×2000 magnification).

Table 1. Types of manufactured polymer samples used to evaluate hemocompatibility

\begin{tabular}{lc}
\hline & Sample type \\
\hline $\mathrm{PHBV} / \mathrm{PCL}$ & Unmodified matrix \\
$\mathrm{PHBV} / \mathrm{PCL} / \mathrm{PVP}$ & Matrix polymerized with polyvinylpyrrolidone \\
$\mathrm{PHBV} / \mathrm{PCL} / \mathrm{PVP} / \mathrm{Ilo} / \mathrm{A}$ & Matrix modified by complexation between polyvinylpyrrolidone and iloprost and amphiphile coating \\
$\mathrm{PHBV} / \mathrm{PCL} / \mathrm{A}$ & Matrix with covalently cross-linked cationic amphiphile
\end{tabular}

Table 2. The degree of hemolysis and maximum aggregation in human blood platelets after their contact with modified and unmodified polymer matrices

\begin{tabular}{lcc}
\hline Sample type & Maximum platelet aggregation, \% Me (25\%-75\%) & The degree of hemolysis, \% Me (25\%-75\%) \\
\hline PHBV/PCL & $87.23(83.95-89.84)^{*}$ & $0.504(0.0-1.01)$ \\
PHBV/PCL/PVP & $88.53(86.59-89.37)^{*}$ & $0.2(0.0-0.5)$ \\
PHBV/PCL/PVP/Ilo/A & $12.18(11.15-12.24)^{*} \#$ & $0.5(0.0-0.5)$ \\
PHBV/PCL/A & $83.28(83.21-84.86)$ & $100(100-100)$
\end{tabular}

Intact platelet-rich plasma (PRP)

$74.65(72.45-75.31)$

* $\mathrm{p}<0.05$ relative to intact $\mathrm{PRP} ; \# \mathrm{p}<0.05$ relative to unmodified $\mathrm{PHBV} / \mathrm{PCL}$ matrices.

Table 3. Platelet adhesion indicators after the contact with PHBV/PCL polymer matrices, depending on the coating type

\begin{tabular}{|c|c|c|c|c|c|c|c|}
\hline \multirow{2}{*}{ Sample type } & \multicolumn{5}{|c|}{ Platelet type, \% } & \multirow{2}{*}{ Platelet count per $\mathrm{mm}^{2} \mathrm{Me}(25-75 \%)$} & \multirow{2}{*}{ Platelet deformation index Me (25-75\%) } \\
\hline & I & II & III & IV & $\mathrm{V}$ & & \\
\hline $\mathrm{PHBV} / \mathrm{PCL}$ & 7.7 & 30.8 & 53.8 & 7.7 & 0.0 & $578.0(0.0-1349.0)$ & $1.75(0.0-2.9)$ \\
\hline PHBV/PCL/PVP & 3.0 & 27.3 & 45.5 & 21.2 & 3.0 & $1156.0(0.0-3082.0) *$ & $1.91(0.0-2.9)$ \\
\hline PHBV/PCL/PVP/Ilo/A & 12.5 & 62.5 & 18.8 & 6.2 & 0.0 & $770.6(0-1445.0)^{\#}$ & $1.3(0.0-2.4)^{\#}$ \\
\hline $\mathrm{PHBV} / \mathrm{PCL} / \mathrm{A}$ & \multicolumn{7}{|c|}{ Platelet count was not possible due to presence of the monolayer of platelets and plasma proteins } \\
\hline
\end{tabular}

* $p<0.05$ relative to the values of the PHBV/PCL matrix indicators; $\# p<0.05$ to the values of the PHBV/PCL/PVP indicators

Table 4. Types of polymer samples used for cytotoxicity analysis

\begin{tabular}{lc}
\hline & Sample type \\
\hline PHBV/PCL & Unmodified matrix \\
$\mathrm{PHBV} / \mathrm{PCL} / \mathrm{PVP} / \mathrm{Ilo}$ & Matrix polymerized with polyvinylpyrrolidone and iloprost \\
$\mathrm{PHBV} / \mathrm{PCL} / \mathrm{PVP} / \mathrm{A}$ & Matrix polymerized with polyvinylpyrrolidone and amphiphile \\
$\mathrm{PHBV} / \mathrm{PCL} / \mathrm{PVP} / \mathrm{Ilo} / \mathrm{A}$ & Matrix polymerized with polyvinylpyrrolidone and iloprost with amphiphile \\
$\mathrm{PHBV} / \mathrm{PCL} / \mathrm{A}$ & Matrix with covalently cross-linked cationic amphiphile \\
\hline
\end{tabular}



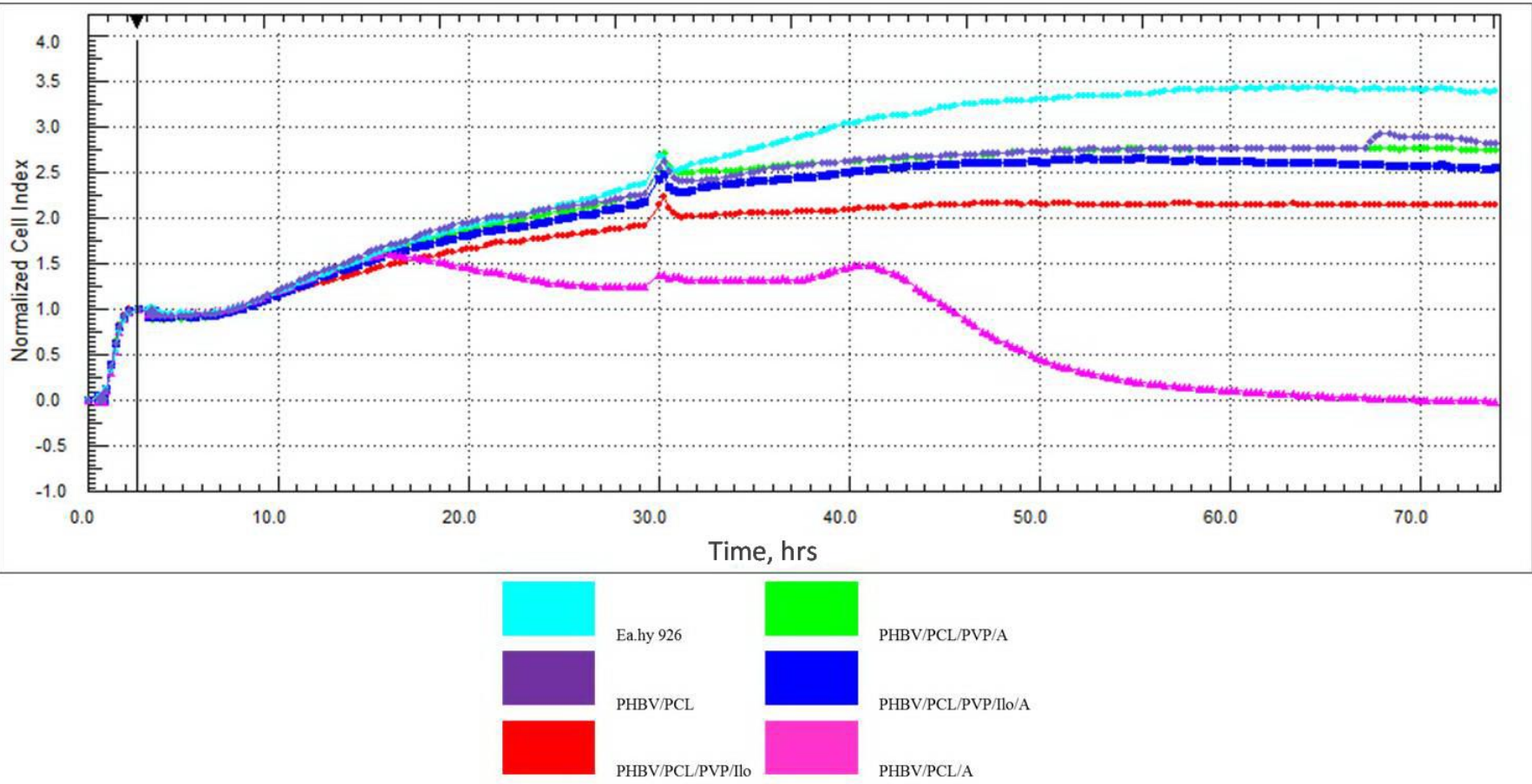

Figure 2. Normalized cell index values of the control and tissue-engineered matrix groups. The point of maximum cell adhesion after seeding them into the wells is assumed to represent the normalization point.
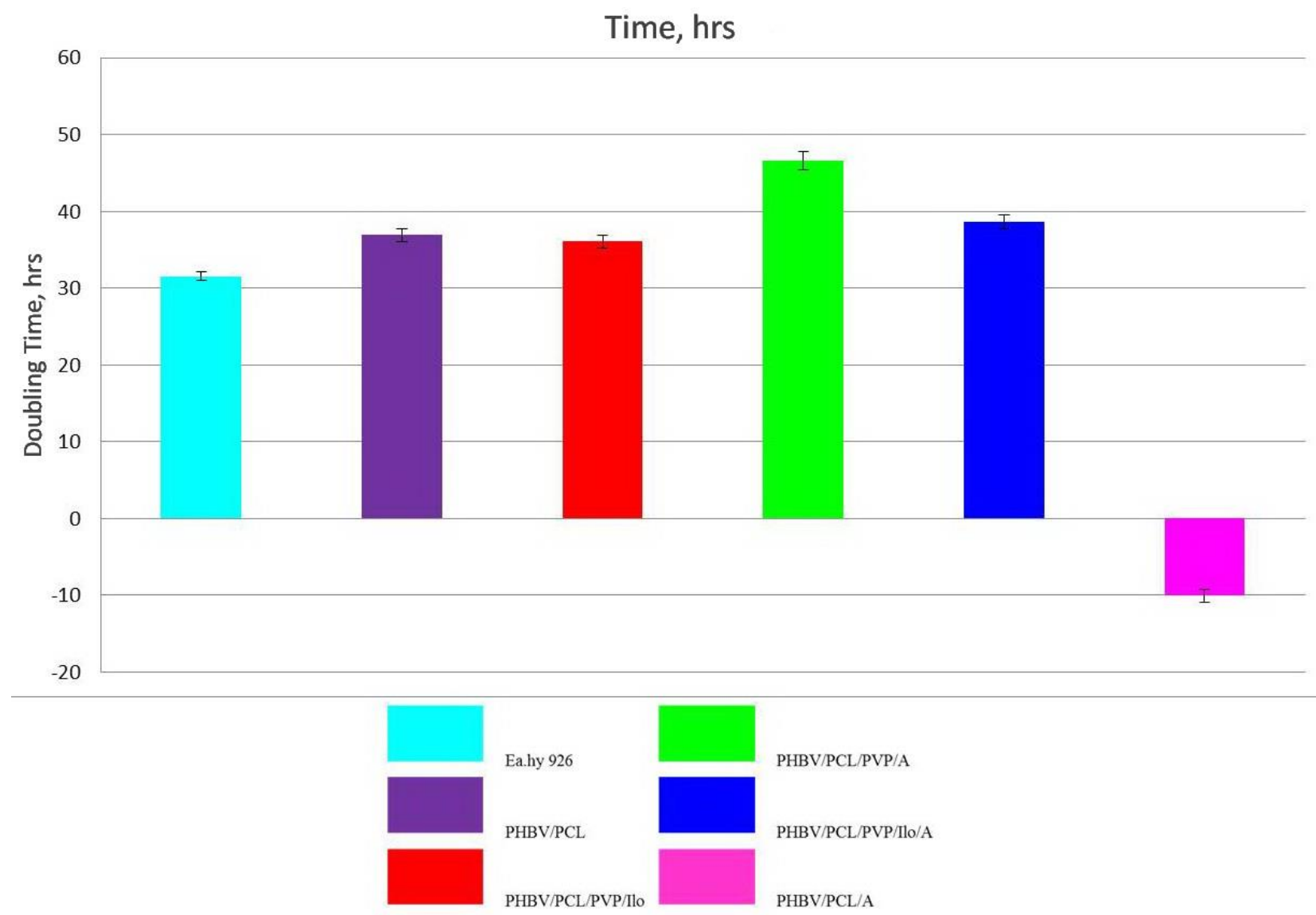

Figure 3. Cell culture doubling time vs. control and tissue-engineered matrix groups. 
Platelet types depending on activation are as follows:

I. Disc-shaped platelets without deformation;

II. Large platelets with pseudopod-shaped protrusions;

III. Large irregularly shaped platelets with pseudopods; they tend to aggregate in conglomerates;

IV. Spreading platelets; their cytoplasm spreads and fills the spaces between pseudopods;

V. Dense granules of platelets; pseudopods cannot be found due to the proliferation of the cytoplasm.

Scanning electron microscopy (SEM) of PHBV/PCL matrices was performed after the contact with PRP.

\section{Assessment of cytotoxicity of tissue-engineered matrix samples with drug coatings}

The effect of test samples on cell vital activity was assessed via the cell index $(\mathrm{Cl})$, measured by the Agilent xCELLigence RTCA DP system (ACEA Biosciences, USA). CI was calculated automatically by the device according to the formula: $(R n-R b) / t$, where $R b$ is the initial resistance value in the well containing the culture medium without cells, $\mathrm{Rn}$ is the resistance value at any time $\mathrm{t}$ in the well containing the cells. The cells were seeded at the density of 20,000 cells per well into an E-Plate 16 (ACEA Biosciences, USA). E-Plate Inserts (ACEA Biosciences, USA) were placed inside the plate wells with the matrix samples. The procedure was repeated at least 2 times for each sample. The wells, containing only cells, were used as a control. The wells filled with culture medium were used as the baseline resistance values.

Cells were incubated in standard DMEM/F12 medium supplemented with $\mathrm{HAT}$, antibiotics/antimycotics in a $\mathrm{CO}_{2}$ incubator at $37{ }^{\circ} \mathrm{C}$ and $5 \% \mathrm{CO}_{2}$.

$\mathrm{Cl}$ was recorded as follows: every $15 \mathrm{~min}$ over the first 2 hours after cell seeding; every $30 \mathrm{sec}$ within 2 hours after the introduction of the tested samples; then, every 15 min until the end of the experiment. The total time of the experiment was 74 hours. The cell index and the cell culture doubling time were calculated on the device automatically, and the data were presented as $\mathrm{M} \pm \mathrm{SD}$.

\section{Statistical analysis}

The obtained data were processed using the GraphPad Prism 7.0 Software (GraphPad, USA). The sample distribution was evaluated using the Kolmogorov-Smirnov test. The results are presented as medians and interquartile ranges (Me; Q1-Q3). Statistically significant differences between two independent groups were assessed via the Mann-Whitney $U$ test. At $p<0.05$, the differences were considered statistically significant.

\section{Results}

\section{Hemocompatibility of modified polymer matrix}

We used the matrices presented in Table 1 to analyze the hemocompatibility of grafts.

\section{Hemolysis}

Hemolysis values did not exceed the norm of $2 \%$ (Table 2). There were no significant differences between the sample groups, with the exception of the surface of PHBV/PCL/A matrices, since the hemolysis there reached $100 \%$.

\section{Platelet aggregation}

Polymerization of the matrix surface with PVP by ionizing radiation increased platelet aggregation by 1.2 times (Table 1 ). However, subsequent complexation between polymerized PVP, and iloprost and amphiphile, resulted in sevenfold reduction in platelet aggregation $(p=0.012)$, compared with intact PRP (Table 2). Such significant decrease in platelet aggregation was apparently associated with iloprost action, since platelet aggregation on PHBV/PCL matrices with covalently cross-linked amphiphile alone did not lead to any aggregation reduction of comparable scale.

\section{Platelet adhesion and platelet deformation index}

PHBV/PCL, matrices polymerized with PVP, demonstrated twofold increase in the platelet adhesion degree $(p=0.0087)$ (Table 3, Figure 1), with a slight increase in the number of type IV platelets. Simultaneously, PDI after polymerization with PVP did not change much. The anti-thrombogenic and antimicrobial Ilo/A coating of PHBV/PCL/PVP matrices induced a 1.5-fold decrease $(p=0.0007)$ in the number of adhered platelets, along with a twofold decrease in the number of type III platelets. Therefore, type I and II platelets became the prevalent types on the surface of the $\mathrm{PHBV} / \mathrm{PCL} / \mathrm{PVP} / \mathrm{Ilo} / \mathrm{A}$ matrices, ultimately causing a 1.5 -fold reduction of $P D I$ value $(p=0.0006)$.

PHBV/PCL matrices with covalently cross-linked amphiphile exhibited a negative effect on platelet adhesion indicators. It was not possible to count the platelets and calculate the deformation index after the contact of PHBV/PCL/A matrices with PRP, due to the presence of a monolayer of platelets and plasma proteins on the surface of these matrices.

\section{Cytotoxicity of polymer matrices with drug coatings}

The results of hemocompatibility analysis accentuated the need to evaluate the effect of iloprost and amphiphile on growth and proliferation of cells. For this purpose, additional groups of polymer matrices with various compositions were manufactured for comparative assessment of cytotoxicity with the Ea.hy 926 control group (Table 4). Iloprost and cationic amphiphile were used either in combination or separately, making it possible to assess the cytotoxicity of each medicamentous coating individually.

The results of evaluating an impact of the studied samples on cell vital activity yielded the standard cell culture growth curve in control wells (Ea.hy 926). At the early stages of cultivation, the cell growth index was comparable to the control group in the experimental wells containing inserts of different polymer matrices. However, after the first day of cultivation, a decline in the slope of the curve was noted, indicating a slight decrease in the rate of cell growth and proliferation (Figure 2).

However, we did not observe the cytotoxic effect in any matrices - either unmodified, or modified by polymerization between PVP, and iloprost and/or cationic amphiphile.

PHBV/PCL/A matrices, modified by covalent crosslinking, significantly differed from other groups. In this group, on the first day of cell cultivation on PHBV/PCL/A matrices, a significant 
reduction in the growth rate of the cell culture was noted. Subsequently, cell deaths and a sharp drop in the cell index value were noted by the end of the study.

This trend was confirmed by the analysis of the cell culture doubling time (Figure 3). The lowest value was observed in the Ea.hy 926 control group: $31.589 \pm 0.5648$ hrs. Most experimental samples were characterized by a slightly higher value of doubling time, ranging from $36.1129 \pm 0.8352 \mathrm{hrs}$. (PHBV/PCL/PVP/A) to $46.6378 \pm 1.1842 \mathrm{hrs}$. (PHBV/PCL/PVP/llo). The negative value of cell culture doubling time $(-10.0602 \pm 0.8438 \mathrm{hrs}$.), signifying the death of the entire cell culture, was noted in the PHBV/PCL/A group, modified with covalent crosslinking.

\section{Discussion}

The bioabsorbable vascular graft with prolonged reabsorption, the polymers of which can be replaced by the patient's own cells and tissues, subsequently forming a new vessel in its place, may serve an excellent replacement for the autologous arteries and veins $[1,19]$. However, highly porous wall of such grafts induces thrombus formation and infection after the implantation [20]. Therefore, an antimicrobial and anti-thrombogenic coating applied to the graft surface should be capable of preventing such complications.

The polymerization of PHBV/PCL matrices performed in our study with PVP by an ionizing radiation at a dosage of $15 \mathrm{kGy}$ made it possible to develop a hydrogel substrate for the medicines, and to fill the pores of a vascular graft, thereby reducing the risk of thrombus formation, and simultaneously sterilizing the samples by radiation. However, our results implied that this procedure led to twofold increase in platelet aggregation and platelet adhesion degree, without increasing PDI and hemolysis values. Further complexation of iloprost and cationic amphiphile helps eliminating the negative effect of PVP crosslinking, reducing the degree of platelets aggregation and adhesion to the baseline values. Modification of the surface by covalently cross-linked amphiphile resulted in $100 \%$ hemolysis, along with immense platelet aggregation and adhesion, making it impossible to estimate the platelet count. The negative effect of the covalent crosslinking technique has been confirmed by the cytotoxicity analysis of these matrices. At the same time, iloprost and cationic amphiphile were not cytotoxic. Furthermore, cell culture death can be explained by the presence of organic matter residues left after the covalent crosslinking.

\section{Conclusion}

Hence, we have demonstrated that iloprost and/or amphiphile coating improve hemocompatibility of polymer grafts without showing any cytotoxic effects. However, cytotoxicity per se could be neutralized by utilizing the complexation technique. The cytotoxicity analysis has revealed that organic substances used in the amphiphile covalent crosslinking usually had negative effects. In conclusion, complexation with polyvinylpyrrolidone as a binding agent can be considered the most suitable procedure for modifying the surfaces of small-diameter bioabsorbable vascular grafts.

\section{Conflict of interest}

The authors declare no conflicts of interest.

\section{Acknowledgments}

This study was funded by the Russian Science Foundation (grant No. 20-15-00075, "Development of a Small-Diameter Biodegradable TissueEngineered Vascular Graft with Anti-Thrombogenic and Antimicrobial Coating").

\section{Ethical approval}

This article does not contain any materials associated with the studies, performed by any of its authors, that involved human participants or animals.

\section{References}

1. Taggart DP. Current status of arterial grafts for coronary artery bypass grafting. Ann Cardiothorac Surg 2013; 2(4): $427-430$ https://doi.org/10.3978/j.issn.2225-319x.2013.07.21.

2. Pelletier-Galarneau M, Juneau D. Vascular graft infection: Improving diagnosis with functional imaging. J Nucl Cardiol 2020. https://doi.org/10.1007/s12350-020-02269-z.

3. Altun G, Pulathan Z, Hemsinli D. Obturator bypass in the treatment of prosthetic graft infection: Classic but still effective. Turk Gogus Kalp Damar Cerrahisi Derg 2018; 26(3): 480-483. https://doi.org/10.5606/tgkdc.dergisi.2018.15744.

4. Gentili A, Di Pumpo M, La Milia DI, Vallone D, Vangi G, Corbo MI et al. A six-year point prevalence survey of healthcare-associated infections in an Italian teaching acute care hospital. Int J Environ Res Public Health 2020; 17(21): 7724. https://doi.org/10.3390/ijerph17217724.

5. Welborn MB, Valentine RJ. Vascular infection. In: Creager MA, Dzau VJ, Loscalzo J, Eds. Vascular Medicine: A companion to Braunwald's heart disease Elsevier, Inc.; 2006: 859-879. https://doi.org/10.1016/B978-07216-0284-4.50067-1.

6. Sousa JV, Antunes L, Mendes C, Marinho A, Gonçalves A, Gonçalves Ó et al. Prosthetic vascular graft infections: A center experience. Angiol Cir Vasc 2014; 10(2): 52-57 https://doi.org/10.1016/S1646706X(14)70050-3.

7. Aslam S, Darouiche RO. Role of antibiofilm-antimicrobial agents in controlling device-related infections. Int J Artif Organs 2011; 34(9): 752-758. https://doi.org/10.5301/ijao.5000024.

8. Ulloa ER, Singh KV, Geriak M, Haddad F, Murray BE, Nizet V, et al. Cefazolin and ertapenem salvage therapy rapidly clears persistent methicillin-susceptible Staphylococcus aureus bacteremia. Clin Infect Dis 2020; 71(6): 1413-1418. https://doi.org/10.1093/cid/ciz995.

9. Staneviciute E, Na'amnih W, Kavaliauskas P, Prakapaite R, Ridziauskas $M$, Kevlicius $L$, et al. New in vitro model evaluating antiseptics' efficacy in biofilm-associated Staphylococcus aureus prosthetic vascular graft infection. J Med Microbiol 2019; 68(3): 432-439. https://doi.org/10.1099/jmm.0.000939.

10. Zhao G, Hochwalt PC, Usui ML, Underwood RA, Singh PK, James GA, et al. Delayed wound healing in diabetic $(\mathrm{db} / \mathrm{db})$ mice with Pseudomonas aeruginosa biofilm challenge: A model for the study of chronic wounds. Wound Repair Regen 2010; 18(5): 467-477. https://doi.org/10.1111/j.1524-475x.2010.00608.x.

11. Ng VW, Chan JM, Sardon H, Ono RJ, García JM, Yang YY, et al. Antimicrobial hydrogels: A new weapon in the arsenal against multidrug-resistant infections. Adv Drug Deliv Rev 2014; 78: 46-62. https://doi.org/10.1016/j.addr.2014.10.028.

12. Ghosh C, Haldar J. Membrane-active small molecules: Designs inspired by antimicrobial peptides. ChemMedChem 2015; 10(10): 1606-1624. https://doi.org/10.1002/cmdc.201500299.

13. Molchanova N, Hansen PR, Franzyk H. Advances in Development of antimicrobial peptidomimetics as potential drugs. Molecules 2017; 22(9): 1430. https://doi.org/10.3390/molecules22091430.

14. Antonova LV, Sevostyanova VV, Rezvova MA, Krivkina EO, Kudryavtseva YA, Barbarash OL, et al. Technology of Producing Functionally Active Biodegradable Small-Diameter Vascular Prostheses 
with Drug Coating. Patent No. 2702239. Registration date: October 07,

2019, Bull. No. $28 . \quad$ Russian. https://www.elibrary.ru/item.asp?id=41276645.

15. Ye X, Wang Z, Zhang X, Zhou M, Cai L. Hemocompatibility research on the micro-structure surface of a bionic heart valve. Biomed Mater Eng 2014; 24(6): 2361-2369. https://doi.org/10.3233/bme-141049.

16. Shen X, Su F, Dong J, Fan Z, Duan Y, Li S. In vitro biocompatibility evaluation of bioresorbable copolymers prepared from L-lactide, 1, 3trimethylene carbonate, and glycolide for cardiovascular applications. J Biomater Sci Polym Ed 2015; 26(8): 497-514. https://doi.org/10.1080/09205063.2015.1030992.

17. Antonova LV, Krivkina EO, Rezvova MA, Sevostyanova VV, Tkachenko VO, Glushkova TV, et al. Technology for anti-thrombogenic drug coating of small-diameter biodegradable vascular prostheses. Sovrem $\begin{array}{llll}\text { Tekhnologii } \quad \text { Med 2020; } & \text { 12(6): } & 6-12\end{array}$ https://doi.org/10.17691/stm2020.12.6.01.

18. Jung $F$, Braune $S$, Lendlein $A$. Haemocompatibility testing of biomaterials using human platelets. Clin Hemorheol Microcirc 2013; 53(1-2): 97-115. https://doi.org/10.3233/ch-2012-1579.

19. Ren $X$, Feng $Y$, Guo J, Wang $H$, Li Q, Yang J, et al. Surface modification and endothelialization of biomaterials as potential scaffolds for vascular tissue engineering applications. Chem Soc Rev 2015; 44(15): 5680-574. https://doi.org/10.1039/c4cs00483c.

20. Antonova LV, Mironov AV, Yuzhalin AE, Krivkina EO, Shabaev AR, Rezvova MA, et al. A brief report on an implantation of small-caliber biodegradable vascular grafts in a carotid artery of the sheep.

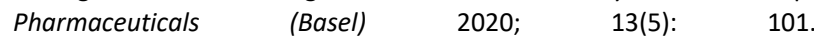
https://doi.org/10.3390/ph13050101.

Authors:

Eugenia 0. Krivkina - Junior Researcher, Laboratory of Cellular Technology, Department of Experimental Medicine, Research Institute for Complex Issues of Cardiovascular Diseases, Kemerovo, Russia. https://orcid.org/0000-0002-2500-2147.

Elena A. Velikanova - PhD, Researcher, Laboratory of Cellular Technology, Department of Experimental Medicine, Research Institute for Complex Issues of Cardiovascular Diseases, Kemerovo, Russia. https://orcid.org/0000-0002-1079-1956.

Eugenia A. Senokosova - PhD, Junior Researcher, Laboratory of Cellular Technology, Department of Experimental Medicine, Research Institute for Complex Issues of Cardiovascular Diseases, Kemerovo, Russia. https://orcid.org/0000-0002-9430-937X.

Maryam Yu. Khanova - Junior Researcher, Laboratory of Cellular Technology, Department of Experimental Medicine, Research Institute for Complex Issues of Cardiovascular Diseases, Kemerovo, Russia. https://orcid.org/0000-0002-8826-9244.

Tatyana V. Glushkova - PhD, Senior Researcher, Laboratory of Novel Biomaterials, Department of Experimental Medicine, Research Institute for Complex Issues of Cardiovascular Diseases, Kemerovo, Russia. https://orcid.org/0000-0003-4890-039.

Larisa V. Antonova - DSc, Head of the Laboratory of Cellular Technology, Department of Experimental Medicine, Research Institute for Complex Issues of Cardiovascular Diseases, Kemerovo, Russia. https://orcid.org/0000-0002-8874-0788.

Leonid S. Barbarash - DSc, Professor, Research Institute for Complex Issues of Cardiovascular Diseases, Academician of the Russian Academy of Sciences, Kemerovo, Russia. https://orcid.org/0000-0002-2814-4300. 